\title{
Laser-induced backward transfer of nanoimprinted polymer elements
}

\author{
Matthias Feinaeugle $^{1}$ (D) Daniel J. Heath ${ }^{1} \cdot$ Benjamin Mills $^{1} \cdot$ James A. Grant-Jacob $^{1}$. \\ Goran Z. Mashanovich ${ }^{1} \cdot$ Robert W. Eason ${ }^{1}$
}

Received: 19 November 2015/Accepted: 29 February 2016/Published online: 15 March 2016

(C) The Author(s) 2016. This article is published with open access at Springerlink.com

\begin{abstract}
Femtosecond laser-induced backward transfer of transparent photopolymers is demonstrated in the solid state, assisted by a digital micromirror spatial light modulator for producing shaped deposits. Through use of an absorbing silicon carrier substrate, we have been able to successfully transfer solid-phase material, with lateral dimensions as small as $\sim 6 \mu \mathrm{m}$. In addition, a carrier of silicon incorporating a photonic waveguide relief structure enables the transfer of imprinted deposits that have been accomplished with surface features exactly complementing those present on the substrate, with an observed minimum feature size of $140 \mathrm{~nm}$.
\end{abstract}

\section{Introduction}

Laser-induced transfer, which relies on the energy of an incident laser pulse to transfer a deposit (or variously termed voxel) of material (the donor) from a carrier substrate towards a receiver substrate, encompasses a range of techniques for rapid microfabrication of electronic, photonic and biomedical devices [1-5]. Recent results have shown the lateral shaping of deposits in a dynamic fashion for laser-induced forward transfer (LIFT), via the use of a digital micromirror device (DMD) acting as a spatial light modulator [6, 7], hence enabling the rapid prototyping of complex shapes with micron-scale fabrication resolution. This approach provides a more flexible alternative to

Matthias Feinaeugle

Matthias.Feinaeugle@ soton.ac.uk

1 Optoelectronics Research Centre, University of Southampton, Highfield Campus, Southampton SO17 1BJ, UK focussing or imaging of an aperture [8,9] and complements alternative beam-shaping approaches previously used for LIFT, which assist in the pre-machining of the donor for transfer of structures with small dimensions [10], smooth side walls [11] or reduced amount of debris [12].

While the ability to shape the deposit in the lateral direction is undoubtedly useful, many applications in electronics and photonics can often require feature sizes down to the nanometre range, far below the optical diffraction limit, and hence cannot be directly achieved via beam shaping. Transfer of nanodroplets can be achieved with the donor in a molten or liquid state [13], although this limits one of the main benefits of laser-induced transfer, namely the possibility of maintaining a previously optimized phase and structure of a thin film.

In this work, we present the results from a different approach whereby the intact transfer of solid deposits has been achieved via laser-induced backward transfer (LIBT), where the deposits produced can have feature sizes well below the diffraction limit. This was achieved via use of a silicon carrier substrate that incorporated a prefabricated photonic waveguide relief structure, so that the donor material was imprinted with the complementary waveguide relief features before the LIBT process. This imprint transfer is a process allowing the fabrication of nanostructures which has not been demonstrated on such a small deposit scale with a direct-write laser technique [14], and such pre-structuring of a voxel prior to transfer could increase the complexity and hence functionality of the printed device. The transfer of solid-phase donors is integral to this technique, as transfer of donors via partial or complete melt or liquid phase does not allow the preservation of the intended surface structure.

In this study, the overall shape (but not the subdiffraction-limited structures) of the deposited material was 
controlled via DMD-based image projection. Conventional lithography or direct exposure of the photoresist on the receiver would not allow such partial structuring of a voxel surface and would not work with photoinsensitive polymer materials. Compared with laser photopolymerization direct-write techniques, the receiving substrate is not exposed to undeveloped liquid resist or chemicals required during development or washing steps. Also, a DMD allows the pixelated transfer of voxels in a single process step and combines other advantages of laser-induced transfer such as high speed, non-contact, the possibility to use non-planar receiver substrates and the lack of required post-processing steps. Using this combination of techniques, we have been able to obtain overall minimum lateral dimensions of a few microns and carrier-imprinted surface feature dimensions of $\sim 140 \mathrm{~nm}$. In this paper, we discuss LIBT in comparison with LIFT and present experimental results for LIBT using unstructured and structured carrier substrates.

\section{Background}

In the LIBT process, the propagation direction of the deposit is typically at an angle of $180^{\circ}$ with respect to the direction of the incident laser pulse (hence the term 'backward'). As shown in Fig. 1a, a small volume from the donor is transferred to the receiving substrate, via absorption of a laser pulse that has been directed through the transparent receiver and donor before being absorbed at the interface of the donor and absorbing carrier. In the literature, LIBT has been used for the deposition of $\mathrm{Bi}_{2} \mathrm{O}_{3}$ [15], for fabricating diffractive structures [16], for plasmonic nanospheres [13] and for liquids aimed at biomedical applications [17], but has been less widely adopted so far than LIFT-based processes and mainly with the donor in a molten or liquid state.

While the release mechanism responsible for LIBT is not the main focus of this work, contributing processes are suspected to be either thermal [18], shock induced via spallation [19], through the vaporization of carrier or donor

(a)

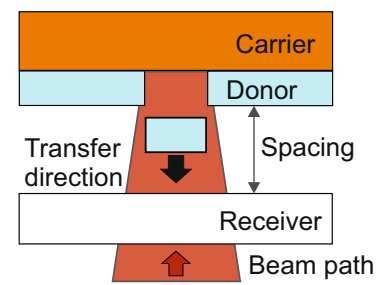

Fig. 1 Schematic of laser-induced backward transfer with a unstructured carrier during LIBT and $\mathbf{b}$ structured carrier after transfer volumes to enable a vapour-driven release of the deposit [20], or via ultrafast expansion if using femtosecond laser pulses [21]. Although we have not yet looked at LIBT with other laser sources (e.g. nanosecond pulse duration), we believe the localization of the laser-induced effects in both space and time that are caused by femtosecond pulse exposure plays an important role in the LIBT mechanism. However, of immediate interest is to briefly compare the two transfer techniques as there is likely an important difference in the absorption profile of the laser energy within the carrier (for LIBT) and at the carrier-donor interface (for LIFT).

In LIBT, reported here, the receiver and donor must be transparent to the incident laser light, which is absorbed in the interfacial volume of the carrier. This requirement would suggest that fabrication of, e.g., photonic or microfluidic devices would be an appropriate end goal for such LIBT-based transfer, due to the possibility to work with flexible and thin polymer donors and receivers. For LIFT of non-absorbing donors, required for a fair comparison of the two techniques, a dynamic release layer (DRL) which is sandwiched between the donor and the carrier needs to be present to absorb the incident laser pulse and initiate the transfer of the donor. In the case of solidphase LIBT, the majority of the laser energy is absorbed in the first $\sim$ few $10 \mathrm{~s}$ of $\mathrm{nm}$ of the carrier and not within the non-absorbing donor. There is therefore a very strong thermal gradient originating at the carrier-donor interface, and this interfacial region of the carrier experiences the largest change in its physical properties which is likely responsible for the subsequent detachment and backward propagation of the donor deposit.

In the case of LIFT, the majority of the laser energy is absorbed within the skin depth of a DRL. In contrast to LIBT, therefore, in LIFT the region of maximum laserinduced change in physical properties occurs on the rear side of the DRL and not on the side facing the donor, and thus, release of the deposit is possibly governed by different physical mechanisms. There is also the additional contamination problem for forward transfer that some DRL material may remain on the released donor deposit or receiver after transfer as a thin film DRL is released or even dissociated easier from the carrier than during LIBT where a bulk carrier substrate is present. This important difference between LIBT and LIFT, we suggest, represents an important factor in the nature of the deposition process which could lead to higher-quality deposition when using LIBT.

In addition, as presented for the first time here, the LIBT technique enables imprinting of the donor via use of a prestructured carrier substrate as shown in Fig. 1b. We do not believe, and to date have not seen within the literature, that this imprinting approach is as practical with LIFT, due to 
the difficulties to fabricate a compliant absorbing layer with constant thickness. Compared with LIFT, LIBT also has the benefit that the absorbing layer can be a bulk substrate, of arbitrary thickness and physical properties, provided it possesses the requisite characteristics to support high absorption of the laser pulse and transfer of the donor.

\section{Experimental}

Experiments used a Ti:sapphire laser oscillator and amplifier chain with a central wavelength of $800 \mathrm{~nm}$ and a pulse duration of $\sim 150 \mathrm{fs}$ (Coherent, Legend) as shown in Fig. 2. The spatial intensity profile of the laser output was homogenized via a refractive top-hat beam shaper (Adloptica, Pi-Shaper) to uniformly illuminate the surface of the DMD array. An object mask displayed on the DMD was then imaged with the combination of a collimating tube lens and an infinity-corrected $20 \times$ or $50 \times$ microscope objective at the sample interface, which was translated on a computer-controlled mechanical stage. Pulse energies were adjusted to investigate the threshold for optimum LIBT, while the sample and image position were monitored with a CMOS camera, a white light source and a dichroic mirror. Further details of this setup can be found in a previous study [7].

Donors were fabricated via spin coating of S1813 and SU-8 photoresists onto cleaned carrier substrates, and baked on a hotplate at $110^{\circ} \mathrm{C}$ for $5 \mathrm{~min}$. The donors were chosen to be materials conventionally used in lithography and served as ideal testbeds for observing achievable resolution via backward transfer. The carrier substrates were polished monocrystalline silicon wafers and silicon-on-insulator chips. The latter consisted of grating structures and slot/rib waveguides and had a layer structure from top to bottom consisting of $\sim 400-\mathrm{nm}$ silicon, $2-\mu \mathrm{m}$ silicon dioxide and $\sim 600-\mu \mathrm{m}$ silicon. These structures were fabricated by e-beam lithography using ZEP e-beam resist for pattern definition and by subsequent transfer to the silicon

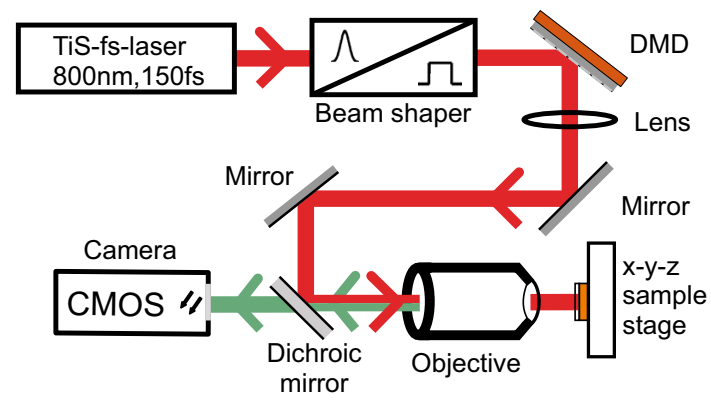

Fig. 2 Setup used for our experiments. DMD, laser source and mechanical sample stage are controlled via computer (not shown) layer using inductively coupled plasma etching. The termination after the etching was native oxide. Receivers tried were bare 1-mm-thick microscope slides, microscope slides coated with $\sim 10-\mu \mathrm{m}$-thick polydimethylsiloxane (PDMS) or free-standing PDMS films, which were chosen as examples for hard, soft and mechanically flexible receivers. The free-standing films were obtained by peeling off the PDMS film from a glass slide.

\section{Results and discussion}

\subsection{LIBT using an unstructured carrier}

Donors (1.4, 1.5, 1.85 and $2.25 \mu \mathrm{m}$ thick) and receivers were in close contact during the experiments, and typical lateral voxel dimensions were in the range of $20-50 \mu \mathrm{m}$. No spacer was used for establishing a defined donor-receiver distance. Due to material inhomogeneities, this spacing was estimated to be in the range of $0-5 \mu \mathrm{m}$, observed from white light thin film interference off the surfaces of donor and receiver. For a fluence just below threshold for transfer, bulging of the donor by some tens of nanometres was observed. The threshold fluence for S1813 for donor thicknesses of 1.4, 1.85 and $2.25 \mu \mathrm{m}$ was measured to be $\sim 475, \sim 580$ and $\sim 765 \mathrm{~mJ} / \mathrm{cm}^{2}$, respectively. As expected, an increased donor thickness required a higher pulse fluence to achieve transfer, and complex shapes with a large ratio of sidewall area to donor-carrier interface (such as the numbers and letters shown in Fig. 3) also required a higher transfer fluence than simple shapes
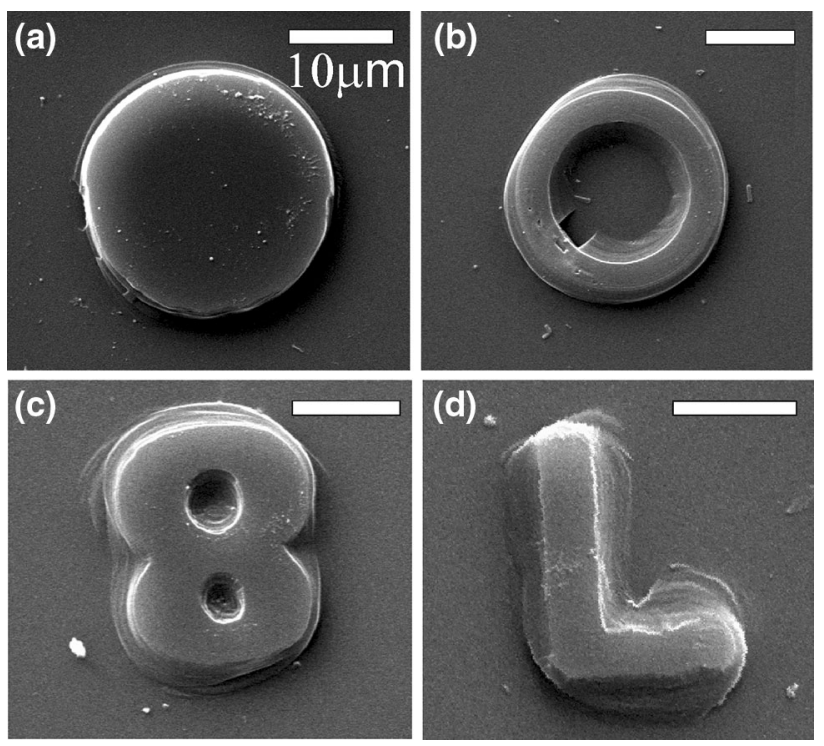

Fig. 3 SEM images of DMD-shaped, $\sim 1.4-\mu$ m-thick S1813 printed via LIBT at a fluence of $\sim 475 \mathrm{~mJ} / \mathrm{cm}^{2}$ (a) and $\sim 510 \mathrm{~mJ} / \mathrm{cm}^{2}$ (bd) onto PDMS-coated glass. The scale bar is $10 \mu \mathrm{m}$ 
such as circles or squares. We note that transfer threshold values were below the damage thresholds of the donors of $\sim 1 \mathrm{~J} / \mathrm{cm}^{2}$ for both SU-8 and S1813.

The results shown in Fig. 3 demonstrate a few examples of the complexity of shapes that can be printed using LIBT via DMD-based image projection. The deposits $(1.4-\mu \mathrm{m}-$ thick S1813) in Fig. 3 had been printed at fluences of $\sim 475 \mathrm{~mJ} / \mathrm{cm}^{2}$ (Fig. 3a) and $\sim 510 \mathrm{~mJ} / \mathrm{cm}^{2}$ (Fig. 3b-d), respectively, onto a PDMS-coated glass receiver from an unstructured silicon carrier. For $1.5-\mu \mathrm{m}$-thick donors of SU-8, this transfer threshold of simple shapes was $\sim 370 \mathrm{~mJ} / \mathrm{cm}^{2}$. We also successfully demonstrated transfer onto free-standing $10-\mu \mathrm{m}$-thick PDMS, which, as it is a flexible substrate, offers the promise of using LIBT to fabricate thin devices and electronic structures on nonplanar surfaces such as optical fibres. The smallest feature size appeared to be limited by the creation of a tapered, angled border, seen quite clearly in Fig. 3d, for example, with a width of $\sim 1$ up to $\sim 3 \mu \mathrm{m}$. Depending on the application, such a feature might limit the resolution or require the use of further donor preparation prior to transfer $[11,12]$.

The smallest features printed from these donors onto PDMS-coated glass were about 5.6- $\mu \mathrm{m}$-wide rectangular structures. Successful transfer of simple shapes, such as squares and circles, was also demonstrated for uncoated glass receivers. However, more complex shapes were not transferred successfully due to the voxels breaking up as a consequence of using a glass receiver. Generally, transfer at a fluence just above threshold was accompanied with minimal debris further limiting the need for post-processing, e.g. the curing of laser-induced interface damage.

Most deposits ( $>90 \%)$ on a PDMS-coated glass receiver remained adhered after submersion in an ultrasonic water bath for 30 min followed by a nitrogen blow dry, but could be removed completely via manual application of an adhesive tape. On uncoated glass, around $70 \%$ of deposits remained intact after the described ultrasonic bath and nitrogen process.

\subsection{LIBT using a structured carrier}

Here, we demonstrate the use of a pre-structured carrier to achieve transfer of complementary features in the donor deposit. The carrier used was a silicon-on-insulator chip containing photonic structures originally used for mid-infrared wavelengths [22], while here they simply served as substrate with precisely defined surface features on the micro- and nanoscale and fabricated with a well-established lithographic technique. The chip layer structure consisted of a sequence of silicon and 2- $\mu$ m-thick buried oxide layers where the chip surface facing the donor film was capped by a layer of 400-nm-thick silicon. Surface

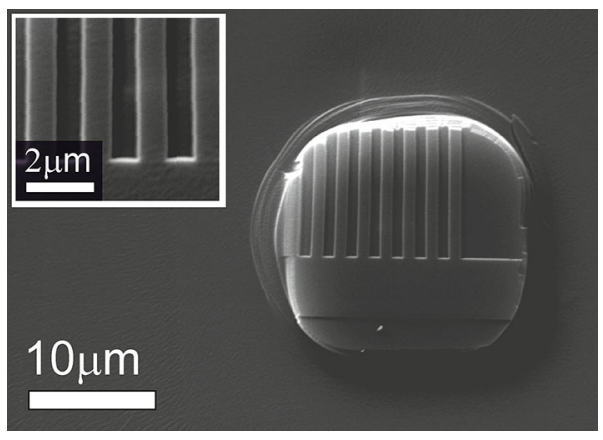

Fig. 4 SEM images of SU-8 LIBT-printed voxel with imprinted grating/waveguide structure on PDMS-coated glass receiver at a fluence of $\sim 475 \mathrm{~mJ} / \mathrm{cm}^{2}$. The inset shows a magnified version of the grating surface with 50/50 mark to space ratio. The resulting finger width and height were $\sim 900$ and $\sim 220 \mathrm{~nm}$, respectively
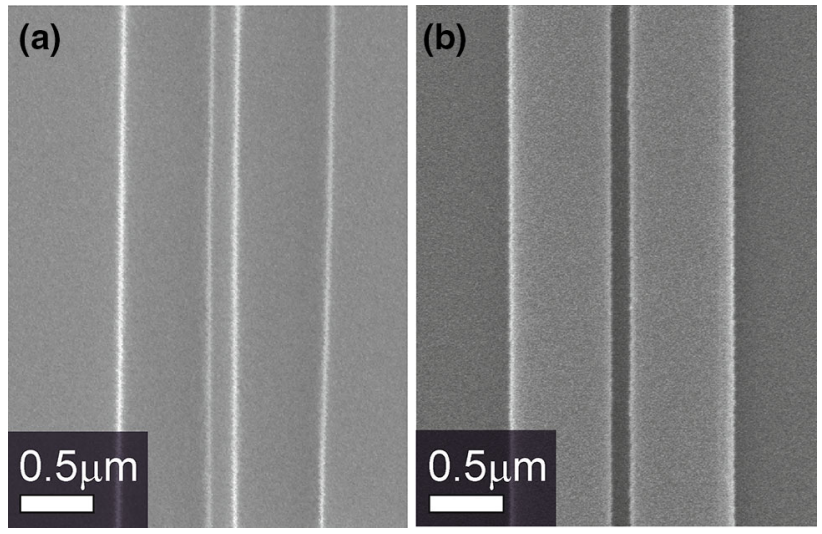

Fig. 5 SEM images of a deposit surface from an S1813 donor printed at $\sim 475 \mathrm{~mJ} / \mathrm{cm}^{2}$ showing a rib within a trench structure and $\mathbf{b}$ a similar slot waveguide structure on a carrier used for the imprinting. The transferred smallest feature on the voxel in the centre of image (a) was $\sim 140 \mathrm{~nm}$ wide and $\sim 220 \mathrm{~nm}$ high

structures had been chemically etched into the top silicon layer prior to LIBT, forming waveguiding and grating relief structures with depths of $\sim 220 \mathrm{~nm}$. During spin coating, the photoresist donor conformed to the carrier in order to create a compliant donor-carrier interface, replicating these relief features with high fidelity, and at the same time forming a smooth and flat layer at the donor-air interface. Results for transfer of an imprint of a grating structure are shown in the SEM image in Fig. 4 for a voxel transferred at a fluence of $\sim 475 \mathrm{~mJ} / \mathrm{cm}^{2}$.

The surface of the deposits on PDMS-coated glass did not show any signs of debris or damage from decomposed donor material, and the grating features are well resolved and have a period of $1.8 \mu \mathrm{m}$ (with 50/50 mark to space ratio).

Results in Fig. 5a show an SEM image of a deposition of an imprinted slot waveguide structure at a surface of a 
circular voxel, transferred at a fluence of $\sim 475 \mathrm{~mJ} / \mathrm{cm}^{2}$, where an SEM image of a similar waveguide structure on the carrier is shown in Fig. 5b. In the deposit, a slot was reproduced as a rib with a width of $\sim 140 \mathrm{~nm}$ for a height of $\sim 220 \mathrm{~nm}$ and centred in a trench of $\sim 1.4 \mu \mathrm{m}$ width.

\section{Conclusion}

In conclusion, we have demonstrated the LIBT of solid photopolymers in an intact state using silicon substrates as absorbing carriers. Spatial voxel shaping was accomplished using a DMD-based image projection system resulting in lateral feature sizes as small as $\sim 6 \mu \mathrm{m}$ for the materials studied. At fluences just above the transfer threshold, the volume of debris and damage to the donor was minimal with reduced edge quality as the interface area was not perfectly sheared from the donor. The limited damage occurring at the interface of carrier and donor and the possibility to use a structured carrier enabled the LIBT technique to be used to reproduce an interfacial carrier relief structure imprinted into the donor layer. This technique therefore enables the simultaneous patterning of the lateral extent and the surface structure of a deposit, with a smallest surface feature size of $\sim 140 \mathrm{~nm}$ enabling applications for electronic or photonic devices.

Acknowledgments This work was funded under the UK Engineering and Physical Sciences Research Council (EPSRC) Grants Nos. EP/L022230/1 and EP/J008052/1. The authors wish to thank Milos Nedeljkovic and Neil Sessions for scientific discussion and technical support. The data for this work are accessible through the University of Southampton Institutional Research Repository (doi:10. 5258/SOTON/381557).

Open Access This article is distributed under the terms of the Creative Commons Attribution 4.0 International License (http://crea tivecommons.org/licenses/by/4.0/), which permits unrestricted use, distribution, and reproduction in any medium, provided you give appropriate credit to the original author(s) and the source, provide a link to the Creative Commons license, and indicate if changes were made.

\section{References}

1. R.S. Braudy, Proc. IEEE 57, 1771 (1969)

2. M.L. Levene, R.D. Scott, B.W. Siryj, Appl. Opt. 9, 2260 (1970)

3. J. Bohandy, B.F. Kim, F.J. Adrian, J. Appl. Phys. 60, 1538 (1986)

4. C.B. Arnold, P. Serra, A. Piqué, MRS Bull. 32, 23 (2007)

5. M. Zenou, A. Sa'ar, Z. Kotler, Sci. Rep. 5, 17265 (2015)

6. R.C.Y. Auyeung, H. Kim, N.A. Charipar, A.J. Birnbaum, S.A. Mathews, A. Piqué, Appl. Phys. A 102, 21 (2011)

7. D.J. Heath, M. Feinaeugle, J.A. Grant-Jacob, B. Mills, R.W. Eason, Opt. Mater. Express 5, 1129 (2015)

8. L. Rapp, C. Constantinescu, Y. Larmande, A.P. Alloncle, P. Delaporte, Appl. Phys. A 117, 333 (2014)

9. M. Feinaeugle, P. Horak, C.L. Sones, T. Lippert, R.W. Eason, Appl. Phys. A 116, 1939 (2014)

10. V. Sametoglu, V. Sauer, Y.Y. Tsui, Appl. Phys. A 110, 823 (2012)

11. K.S. Kaur, M. Feinaeugle, D.P. Banks, J.Y. Ou, F. Di Pietrantonio, E. Verona, C.L. Sones, R.W. Eason, Appl. Surf. Sci. 257, $6650(2011)$

12. R. Pohl, M. Jansink, G.R.B.E. Römer, A.J. Huis in 't Veld, Appl. Phys. A 120, 427 (2015)

13. A.I. Kuznetsov, A.B. Evlyukhin, C. Reinhardt, A. Seidel, R. Kiyan, W. Cheng, A. Ovsianikov, B.N. Chichkov, J. Opt. Soc. Am. B 26, B130 (2009)

14. S.Y. Chou, P.R. Krauss, P.J. Renstrom, Appl. Phys. Lett. 67, 3114 (1995)

15. H. Sakata, S. Chakraborty, M. Wakaki, Microelectron. Eng. 96, 56 (2012)

16. P. Papakonstantinou, N.A. Vainos, C. Fotakis, Appl. Surf. Sci. 151, 159 (1999)

17. A. Patrascioiu, M. Duocastella, J.M. Fernández-Pradas, J.L. Morenza, P. Serra, Appl. Surf. Sci. 257, 5190 (2011)

18. Y.P. Meshcheryakov, M.V. Shugaev, T. Mattle, T. Lippert, N.M. Bulgakova, Appl. Phys. A 113, 521 (2013)

19. S.G. Koulikov, D.D. Dlott, J. Photochem. Photobiol. A. Chem. 145, 183 (2001)

20. N.T. Kattamis, M.S. Brown, C.B. Arnold, J. Mater. Res. 26, 2438 (2011)

21. J. Sotrop, A. Kersch, M. Domke, G. Heise, H.P. Huber, Appl. Phys. A 113, 397 (2013)

22. M. Nedeljkovic, A.Z. Khokhar, Y. Hu, X. Chen, J.S. Penades, S. Stankovic, H.M.H. Chong, D.J. Thomson, F.Y. Gardes, G.T. Reed, G.Z. Mashanovich, Opt. Mater. Express 3, 1205 (2013) 